\title{
Integrin Dynamics Produce a Delayed Stage of Long-Term Potentiation and Memory Consolidation
}

\author{
Alex H. Babayan, ${ }^{1 \star}$ Enikö A. Kramár, ${ }^{1 \star}$ Ruth M. Barrett, ${ }^{2}$ Matiar Jafari, ${ }^{1}$ Jakob Häettig, ${ }^{2}$ Lulu Y. Chen, ${ }^{1}$ \\ Christopher S. Rex, ${ }^{1}$ Julie C. Lauterborn, ${ }^{1}$ Marcelo A. Wood, ${ }^{2}$ Christine M. Gall, ${ }^{1,2}$ and Gary Lynch ${ }^{1,3}$ \\ Departments of ${ }^{1}$ Anatomy and Neurobiology, ${ }^{2}$ Neurobiology and Behavior, and ${ }^{3}$ Psychiatry and Human Behavior, University of California, Irvine, \\ California 92697
}

\begin{abstract}
Memory consolidation theory posits that newly acquired information passes through a series of stabilization steps before being firmly encoded. We report here that in rat and mouse, hippocampus cell adhesion receptors belonging to the $\beta 1$-integrin family exhibit dynamic properties in adult synapses and that these contribute importantly to a previously unidentified stage of consolidation. Quantitative dual immunofluorescence microscopy showed that induction of long-term potentiation (LTP) by theta burst stimulation (TBS) activates $\beta 1$ integrins, and integrin-signaling kinases, at spine synapses in adult hippocampal slices. Neutralizing antisera selective for $\beta 1$ integrins blocked these effects. TBS-induced integrin activation was brief $(<7 \mathrm{~min}$ ) and followed by an $\sim 45 \mathrm{~min}$ period during which the adhesion receptors did not respond to a second application of TBS. Brefeldin A, which blocks integrin trafficking to the plasma membrane, prevented the delayed recovery of integrin responses to TBS. $\beta 1$ integrin-neutralizing antisera erased LTP when applied during, but not after, the return of integrin responsivity. Similarly, infusions of anti- $\beta 1$ into rostral mouse hippocampus blocked formation of long-term, object location memory when started $20 \mathrm{~min}$ after learning but not $40 \mathrm{~min}$ later. The finding that $\beta 1$ integrin neutralization was effective in the same time window for slice and behavioral experiments strongly suggests that integrin recovery triggers a temporally discrete, previously undetected second stage of consolidation for both LTP and memory.
\end{abstract}

\section{Introduction}

The commonplace observation that a blow to the head causes forgetting of recent, but not temporally distant, events led 19th century researchers to posit that memories are progressively stabilized over the several minutes following initial learning (Ribot, 1882; Müller and Pilzecker, 1900). Subsequent laboratory work revealed that multiple consolidation steps are required before information is firmly encoded. Identifying neurobiological substrates for these stages has since been a primary goal of memory research. Evidence that long-term potentiation (LTP), a form of plasticity closely related to memory, requires rapid (cytoskeletal; Kramár et al., 2006; Lynch et al., 2007; Rex et al., 2009) and delayed (protein synthesis; Bramham, 2008) stabilization processes provided plausible substrates for a strong version of the serial consolidation hypothesis (McGaugh, 2000). The present results call for significant revisions to the argument by describing a previously undetected integrin-dependent stabilization step,

Received April 14, 2012; revised July 5, 2012; accepted July 12, 2012.

Author contributions: A.H.B., E.A.K., R.M.B., M.A.W., C.M.G., and G.L. designed research; A.H.B., E.A.K., R.M.B., M.J., J.H., L.Y.C., and C.S.R. performed research; J.C.L. contributed unpublished reagents/analytic tools; A.H.B., E.A.K., R.M.B., J.H., J.C.L., M.A.W., C.M.G., and G.L. analyzed data; A.H.B., E.A.K., R.M.B., M.A.W., C.M.G., and G.L. wrote the paper.

This work was supported, in part, by NINDS Grant NS045260 to G.L. and C.M.G., NIMH Grants MH082042 to C.M.G. and MH081004 to M.A.W., and NIMH fellowships MH083396 to L.Y.C. and MH85494 to R.M.B.

*A.H.B. and E.A.K. contributed equally to this work.

Correspondence should be addressed to Dr. Gary Lynch, Department of Psychiatry and Human Behavior, Gillespie Neuroscience Research Facility, University of California, Irvine, CA 92697-4291. E-mail: glynch@uci.edu.

DOI:10.1523/JNEUROSCI.2024-12.2012

Copyright $\odot 2012$ the authors $\quad 0270-6474 / 12 / 3212854-08 \$ 15.00 / 0$ occurring between the rapid and late phases, that is critical to both LTP and long-term memory.

Diverse lines of evidence indicate that synaptic adhesion receptors belonging to the $\beta 1$ integrin family are critically involved in the rapid phase of LTP consolidation. Blocking the receptors with ligand-mimetic peptides, selective toxins, genetic manipulations, or neutralizing antisera has little effect on the induction and expression of LTP but nonetheless causes potentiation to decay back to baseline (Chun et al., 2001; Kramár et al., 2002, 2006; Chan et al., 2006, 2010; Huang et al., 2006). Studies using $\beta 1$ integrin-neutralizing antisera also identified a likely route whereby these receptors stabilize LTP: treatments applied before or soon after induction prevent the activity-driven actin polymerization (Kramár et al., 2006) known from a variety of studies to be required for the maintenance of potentiation (Krucker et al., 2000; Fukazawa et al., 2003; Rex et al., 2010). The latter observation is consistent with the potent influence of integrins on cytoskeletal organization at various types of adhesion junctions (Brakebusch and Fässler, 2003). Importantly, treatments that selectively disrupt LTP-related actin filament assembly suppress memory encoding (Rex et al., 2010; Lamprecht, 2011; Motanis and Maroun, 2012).

The above studies firmly connect integrins to rapid consolidation but do not address the question of whether the role played by these receptors is constitutive in nature or involves the dynamic properties found in motile and developing cells (Scales and Parsons, 2011). Integrin activation involves conformational changes that increase affinity for extracellular matrix ligands (Luque et al., 1996), trigger the assembly of a complex aggregate 
of enzymes and adapter proteins at the receptor's cytoplasmic tail (Legate and Fässler, 2009), and stimulate integrin-signaling kinases (Mitra and Schlaepfer, 2006). There is no evidence that comparable dynamics are set in motion by LTP, or indeed occur at mature junctions in stationary cells. We therefore tested whether key steps in integrin activation and signaling are initiated at hippocampal synapses by the induction of LTP. Results confirmed this point, but also showed that integrin activation within adult synapses has dynamic properties not reported for other types of cell adhesion junctions, and that these temporally extended effects result in a delayed phase of LTP and memory consolidation.

\section{Materials and Methods}

Slice preparation and electrophysiology. Acute transverse hippocampal slices $(350 \mu \mathrm{m})$ were prepared from young adult (30- to 60-d-old) male Sprague Dawley rats using a Leica Vibroslicer (VT 1000S) or McIlwain tissue chopper, as described previously (Kramár et al., 2002, 2006; Chen et al., 2007). Slices were cut into ice-cold, high-magnesium, artificial CSF (ACSF) containing (in mM) $124 \mathrm{NaCl}, 3 \mathrm{KCl}, 1.25 \mathrm{KH}_{2} \mathrm{PO}_{4}, 5 \mathrm{MgSO}_{4}, 26$ $\mathrm{NaHCO}_{3}$, and 10 dextrose. Slices were then transferred to an interface recording chamber maintained at $31 \pm 1^{\circ} \mathrm{C}$, oxygenated in $95 \% \mathrm{O}_{2} / 5 \%$ $\mathrm{CO}_{2}$ and constantly perfused $(60-80 \mathrm{ml} / \mathrm{h})$ with normal ACSF (in mM: $124 \mathrm{NaCl}, 3 \mathrm{KCl}, 1.25 \mathrm{KH}_{2} \mathrm{PO}_{4}, 1.5 \mathrm{MgSO}_{4}, 2.5 \mathrm{CaCl}_{2}, 26 \mathrm{NaHCO}_{3}$, and 10 dextrose). Slices equilibrated in the chamber for $\sim 2 \mathrm{~h}$ before experimental use. Bipolar stimulating electrodes (twisted nichrome wire, 65 $\mu \mathrm{m}$ ) were placed in CAla and CA1c to stimulate distinct populations of Schaffer-collateral projections to elicit field EPSPs (fEPSPs) in CA1b stratum (str.) radiatum. After stable baseline fEPSP responses were obtained (10 $\mathrm{min}$ of $3 / \mathrm{min}$ stimulation), an input (stimulus intensity)/ output (fEPSP slope) curve was established to determine the overall health of the slice. A new baseline was established over 20 min with stimuli delivered to elicit responses at $40-50 \%$ of the maximum, population spike-free amplitude. Theta burst stimulation (a single train of 10 bursts of 4 pulses at $100 \mathrm{~Hz}$, with $200 \mathrm{~ms}$ between bursts) was then applied, after which responses to 3 pulse/min stimulation were continuously recorded until slice harvest. The stimulus pulse duration was doubled during theta burst stimulation (TBS); control slices received 3/min (baseline-rate) stimulation at the same pulse duration. The fEPSP slope and amplitude were measured using traces digitized by NacGather 2.0. Slices were harvested into $4 \%$ paraformaldehyde in $0.1 \mathrm{~m}$ phosphate buffer, $\mathrm{pH} 7.4$, at $4^{\circ} \mathrm{C}$.

Antisera infusion. Function blocking antisera to $\beta 1$ integrin (MAB1987Z, Millipore; Kramár et al., 2006) and control anti-mouse IgG (\#PP100, Millipore) were diluted to a working pipette concentration of $0.2 \mathrm{mg} / \mathrm{ml}$ in ACSF immediately before use. The IgGs were locally applied by pressure ejection from a pulled glass pipette positioned in the same lamina as the recording electrode (Kramár et al., 2002). Phenol red dye was included in the working solution to follow its diffusion into the slice.

Drug application. Brefeldin A and anisomycin (Tocris Bioscience) were dissolved in dimethyl sulfoxide and later diluted into ACSF for final working concentrations of 35 and $10 \mu \mathrm{M}$, respectively (dimethyl sulfoxide $<0.01 \%$ final).

Immunocytochemistry. Slices were sectioned at $25 \mu \mathrm{m}$ and processed for double-labeling immunofluorescence (Chen et al., 2007, 2010a) using a mixture of primary antisera to the target antigen and to postsynaptic density-95 (PSD-95), a spine synapse marker. The primary antisera mixture consisted of mouse anti-activated $\beta 1$ integrin (1:250; \#MAB2079Z, Millipore; Luque et al., 1996), rabbit anti-phosphorylated (p) Focal Adhesion Kinase (FAK) Tyr397 (1:500, Ab24781, Abcam), or rabbit anti-p-Proline-rich tyrosine kinase 2 (pPyk2) Tyr402 (1:200, 44618G, Biosource; Bernard-Trifilo et al., 2005), in combination with goat anti-PSD-95 (1:100, Santa Cruz Biotechnology) or mouse anti-PSD-95 (1:1000; \#MA1-045, Affinity Bioreagents). Following primary antisera incubation for $18-24 \mathrm{~h}$ at room temperature, the tissue was rinsed and then incubated in the secondary antisera mixture including Alexa Fluor 594 anti-rabbit IgG or Alexa Fluor 594 anti-goat IgG with Alexa Fluor 488 anti-mouse IgG (1:1000 each).
Quantitative analysis of immunostaining. Immunolabeled tissue was imaged using a Leica DM6000 epifluorescence microscope equipped with an Orca-ER charged coupled device camera (Hamamatsu). Individual image $Z$-stacks were collected at $63 \times$ (Plan-Apochromat, numerical aperture 1.4) from the CA1 str. radiatum field of afferent stimulation for the 4-6 uppermost tissue sections from each hippocampal slice. The sample field encompassed $136 \mu \mathrm{m} \times 105 \mu \mathrm{m}$ in the X-Y plane, between the two stimulating electrodes and $Z$-stacks were collected across a depth of $3 \mu \mathrm{m}$ at $0.2 \mu \mathrm{m}$ steps. Images were acquired and processed for iterative deconvolution (99\% confidence) using Volocity 4.0 (PerkinElmer) software; point spread functions were calculated from Multispeck multispectral microspheres $(0.5 \mu \mathrm{m}$, Invitrogen; Rex et al., 2009; Chen et al., 2010c). Quantitative analysis of immunolabeling was conducted as in prior work (Rex et al., 2009; Chen et al., 2010c) with some modifications. Images (8-bit) were normalized for density across the $\mathrm{X}-\mathrm{Y}$ plane by subtracting the low-frequency component to remove nonspecific density irregularities: target background intensities were set to $15 \%$ of the maximal value (Matlab 7, MathWorks). For segmentation, images were iteratively binarized through a fixed interval threshold series ranging from 39 to $78 \%$ of maximum and reconstructed in 3 dimensions (3D). This method provides identification of both faintly and densely labeled elements. Duplicate instances across intervals were compared and best fit was determined as described previously (Rex et al., 2009; Chen et al., 2010c). Immunolabeled object quantification was completed using inclusion criteria based on object size and sphericity. Each 3D montage was analyzed independently for quantification of single- and doubleimmunolabeled elements within the size constraints of dendritic spines; objects were considered double-labeled if there was any overlap in the boundaries of puncta labeled with the two different fluorescent markers. Measures were collected across several tissue sections from each hippocampal slice to determine the mean number of puncta per section for that slice; measures from the different slices from each treatment group were then used to determine the group mean \pm SEM values presented in the text and illustrations.

Analysis of triple immunolabeling. Confocal images were acquired on a LSM 710 NLO laser scanning confocal microscope fitted with a $63 \times$ oil objective. Alexa Fluors were excited at 405, 488, and $594 \mathrm{~nm}$. To ensure no cross talk, the emitted light was collected at 390-415, 480-505, and 575-600 $\mathrm{nm}$, respectively; each channel was acquired separately. All confocal images were acquired at the Nyquist rate to ensure no under-sampling. Original images were deconvolved in Volocity 4.0. All post-acquisition processing and analysis was then performed in Imaris (Bitplane Inc).

Object recognition learning. Training and testing for locationdependent object recognition was performed as described previously (Barrett et al., 2011; Haettig et al., 2011). Before training, 8- to 10-weekold male C57BL6J mice were handled 1-2 min for $5 \mathrm{~d}$ and then habituated to the experimental apparatus (white rectangular open field, $30 \times$ $23 \times 21.5 \mathrm{~cm}) 5 \mathrm{~min}$ a day for 4 consecutive days in the absence of objects. During the training period, mice were placed into the experimental apparatus with two identical objects ( $100 \mathrm{ml}$ beakers) and were allowed to explore for $10 \mathrm{~min}$ (Barrett et al., 2011). During the retention test, $24 \mathrm{~h}$ after training, mice explored the experimental apparatus for $5 \mathrm{~min}$. To examine location-dependent object recognition memory, one copy of the familiar object was placed in the same location as during the training trial and one copy of the familiar object was placed in the middle of the box. All training and testing trials were videotaped and analyzed (by individuals blind to the treatment condition) to determine the amount of time the mouse spent exploring the objects. A mouse was scored as exploring an object when its head was oriented toward the object within a distance of $1 \mathrm{~cm}$ or when the nose was touching the object. The relative exploration time was recorded and expressed by a Discrimination Index $\left(\mathrm{DI}=\left(t_{\text {novel }}-t_{\text {familiar }}\right) /\left(t_{\text {novel }}+t_{\text {familiar }}\right) \times 100 \%\right)$. Mean exploration times were calculated and the discrimination indices between treatment groups at each time point were compared.

Cannulations and injections. Cannulations were performed as described previously (Vecsey et al., 2007). Bilateral 22 gauge cannulae were used to guide injection cannulae (28 gauge) into the dorsal hippocampus (Plastics One Inc.). The guide cannula placement was as follows: anteroposterior, $-1.7 \mathrm{~mm}$; mediolateral, $\pm 1.2 \mathrm{~mm}$; dorsoventral, $1.5 \mathrm{~mm}$ be- 

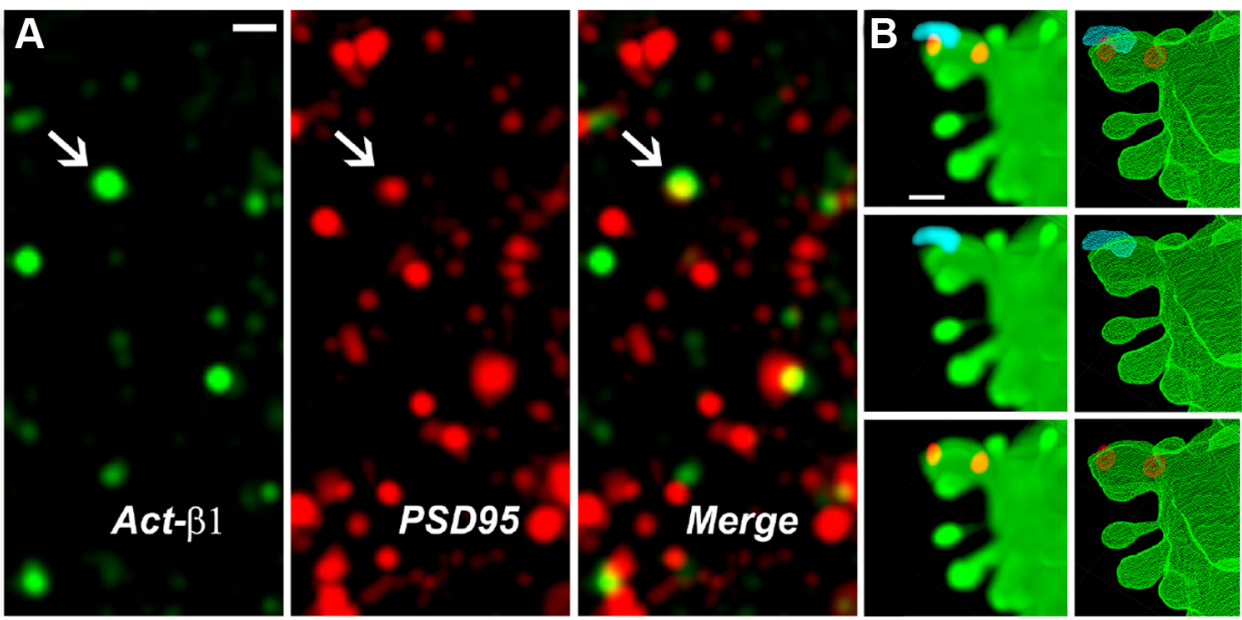

\section{Double-labeled synapses}
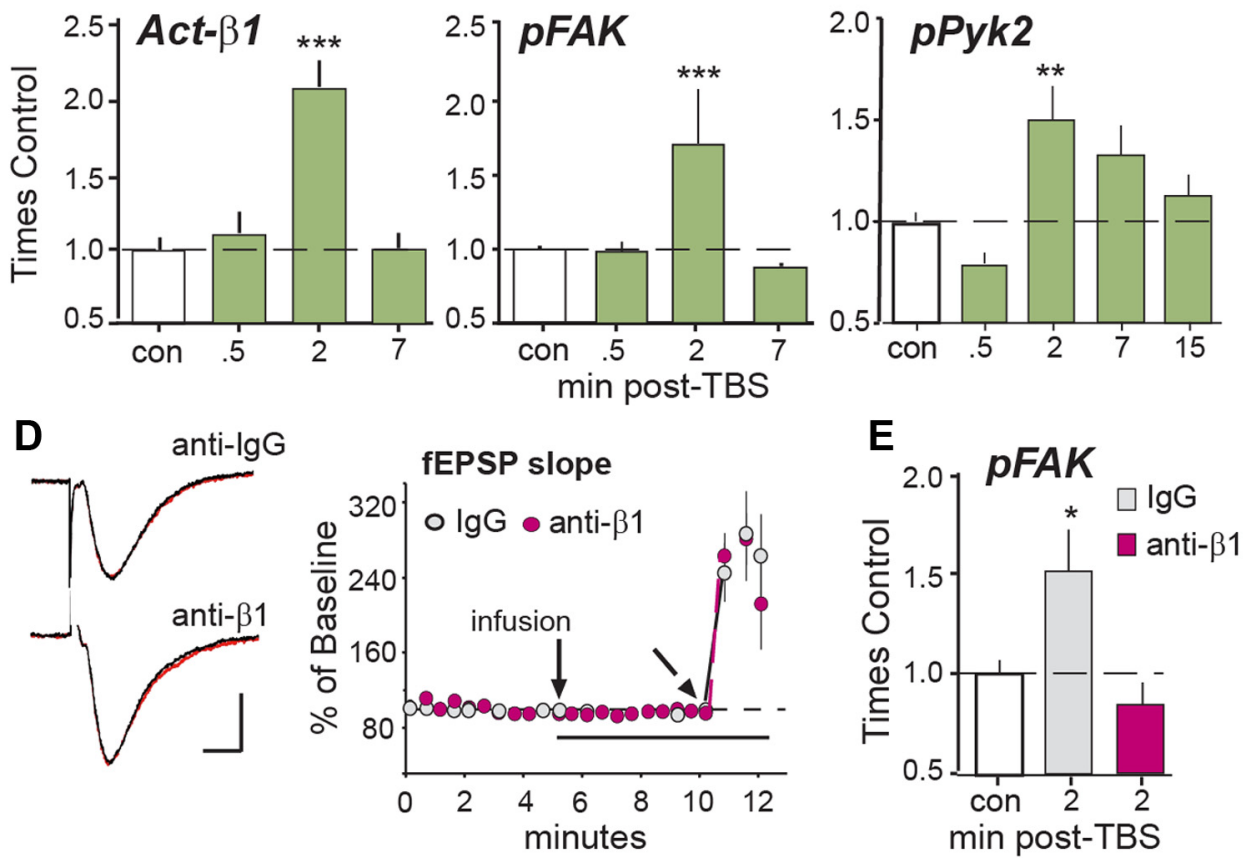

Figure 1. TBS activates synaptic $\beta 1$ integrins. $A$, Deconvolved images show localization of immunoreactivities for activated (Act)- $\beta 1$ and PSD-95 alone, and merged, in field CA1b str. radiatum (bar = $1 \mu \mathrm{m}$ ); arrows indicate a double-labeled synapse. $\boldsymbol{B}$, Immunolocalization of Act- $\beta 1$ (blue) and pFAK (red) on a span of GFP-labeled dendrite from field CA1 of a mouse slice. The maximum intensity projection (left) and 3D reconstruction from confocal image Z-stack (right) shows localization of Act- $\beta 1$ and its signaling kinase on the head of an individual dendritic spine. $C$, Graphs show numbers of PSD-95-immunopositive (+) synapses double-labeled for Act- $\beta 1$, pFAK Tyr397 or pPyk2 Tyr402 (normalized to mean control-slice values) are transiently increased after TBS in the CA1b sample field and peak at the 2 min time point (Act- $\beta 1$ and pFAK ANOVA: $p<0.0001 ;{ }^{* * *} p<0.001$ vs con; pPyk2 ANOVA: $p<0.0003$; ${ }^{* *} p<0.01$ vs con; $n=6-12$ slices/group). $D$, Acute, topical infusions of neutralizing anti- $\beta 1$, or control lgG, have no effect on baseline fEPSPs or initial potentiation following TBS. Left, Representative traces collected during baseline recordings (black) and 5 min after infusion of lgG or anti- $\beta 1$ (red; bars: $1 \mathrm{mV}, 5 \mathrm{~ms}$ ). Right, Plot of fEPSP slopes collected over time (TBS applied at angled arrow). $\boldsymbol{E}$, The same anti- $\beta 1$ treatment completely suppressed TBS-induced increases in synaptic pFAK ( ${ }^{*} p<0.05$ vs con; $p>0.05$ for anti- $\beta 1$ group vs con, Student's $t$ test; $n=12$ slices/group).

low pedestal. Injection cannulae extended an additional $0.5 \mathrm{~mm}$ below the guide cannulae (total depth $2.0 \mathrm{~mm}$ below pedestal). Mice received bilateral intrahippocampal injections of $1 \mu \mathrm{l}$ of $\beta 1$ integrin-neutralizing antisera (MAB1987Z, Millipore; Bernard-Trifilo et al., 2005; Kramár et al., 2006) or control anti-mouse IgG (\#PP100, Millipore; diluted to a working concentration of $0.2 \mathrm{mg} / \mathrm{ml}$ ) per side from a $5.0 \mu$ l Hamilton syringe operated by a Harvard Apparatus Pump II Dual Syringe micropump; mice treated with $\operatorname{IgG}$ and anti- $\beta 1$ at a given time point were processed in the same cohort. Injections occurred over $1 \mathrm{~min}$ and injection cannulae were left in place an additional $30 \mathrm{~s}$ to allow the fluid to diffuse. Each side was injected individually, one immediately after the other. The entire injection process took $\sim 4 \mathrm{~min}$.

Statistical analysis. Statistical significance was determined using either the Student's $t$ test, one-sample $t$ test, or one-way ANOVA. Nonparamet- ric post hoc analysis was conducted using the Kruskal-Wallis (K-W) test. Throughout, $p \leq 0.05$ was considered significant. Group measures presented in the text are mean \pm SEM values.

\section{Results}

Induction of LTP triggers integrin activation and signaling To test whether TBS used to induce LTP in rat hippocampal slices activates synaptic integrins, we used immunolabeling with conformation-specific antisera to the activated $\beta 1$ integrin subunit (Luque et al., 1996; Oinuma et al., 2006). Field EPSPs were elicited by stimulation of the Schaffer-commissural projections and recorded in CAlb throughout the experiments. Double immunolabeling with antisera to the integral synaptic protein 

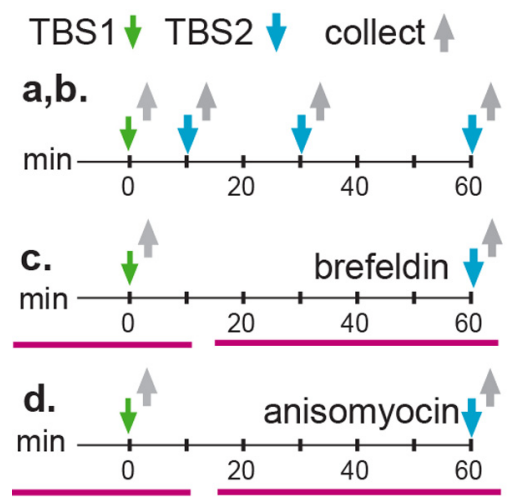

A

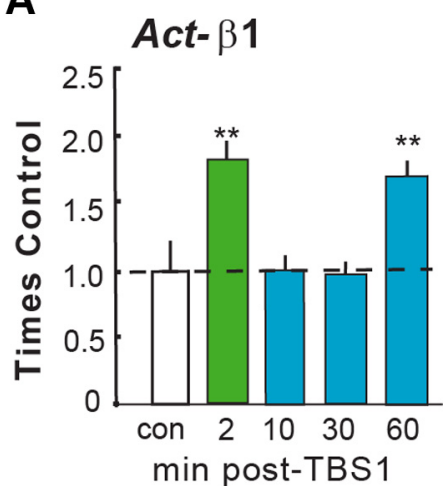

B

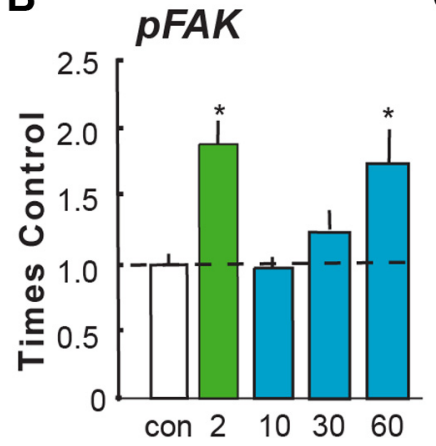

C

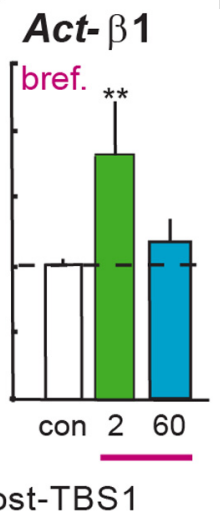

D

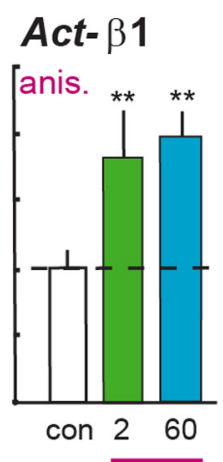

$\min$ post-TBS1

Figure 2. Loss and recovery of integrin signaling. Time lines at top left summarize the experimental designs (time lines and associated results are denoted with lower and upper case letters, respectively). $\boldsymbol{A}, \boldsymbol{B}$, TBS was delivered twice (TBS1, TBS2) with different between-train intervals; slices were collected 2 min after the last TBS and processed for immunofluorescence. $A$, A single TBS train (green bar) increased numbers of PSD-95 + synapses containing immunoreactivity for Act- $\beta 1$ integrin relative to counts from yoked control (con) slices given low-frequency (baseline) stimulation only. TBS2 had no effect on numbers of Act- $\beta 1+$ synapses when delayed by 10 or 30 min after TBS1 but caused the normal increase in Act- $\beta 1+$ synapses at 60 min (blue bars). $\boldsymbol{B}$, Similar effects were obtained for TBS-induced increases in synaptic pFAK. C, Groups of slices were perfused (horizontal red bars in c) for 40 min with brefeldin before TBS1 or TBS2. Brefeldin had no effect on integrin activation by TBS1 but blocked that produced by TBS2. $\boldsymbol{D}$, The design was the same as in $\boldsymbol{C}$ except that slices were treated with anisomycin. The anisomycin infusion had no effect on $\beta 1$ activation by TBS1 or TBS2. ANOVA: $p<0.001$ for all graphs; ${ }^{*} p<0.05$ and ${ }^{* *} p<0.01$ vs con; $\geq 6$ slices/group. driven increases in synaptic pFAK $(86 \pm$ $23 \%$ ) at 2 min post-TBS (Fig. 1E). These findings show that naturalistic patterns of afferent stimulation induce integrin activation and signaling in adult synapses.

\section{Synaptic integrins enter a prolonged refractory period following initial activation}

The transient nature of integrin activation and signaling after TBS made it possible to ask whether one episode of integrin engagement affects subsequent activation. In adult hippocampal slices, TBS was applied as above (TBS1) and then a second TBS train (TBS2) was delivered to the same set of Schaffer-commissural fibers 10,30 , or 60 min later; the slices were collected 2 min after TBS2, the latency at which the activated synapses normally show the greatest increase in activated integrins and pFAK (Fig. 1C). One TBS train increased the number of Act- $\beta 1+$ synapses by the same magnitude as described above. However, TBS2 had no detectable effect on numbers of Act- $\beta 1+$ synapses when applied 10 or 30 min after the first round of stimulation; numbers of PSD-95+ profiles associated with dense concentrations of Act- $\beta 1$ at 2 min after TBS2 were $100 \pm 15 \%$ (10 min delay) and $96 \pm 10 \%$ (30 min delay) of control values (Fig. 2A). This loss of integrin responsiveness to stimulation was transient as evidenced by the effects of a TBS2 train delayed by $60 \mathrm{~min}$; this caused an increase in Act- $\beta 1+$ postsynaptic densities (172 \pm $11 \%$ of control values) which was comparable to that seen 2 min after TBS1. These results provide evidence that synaptic PSD-95 showed that only a small percentage of PSD-95immunopositive $(+)$ synapses were associated with dense labeling for activated $\beta 1$ integrin (Act- $\beta 1$ ) in control slices $(\sim 4 \%$; Fig. $1 A)$. Once activated, integrins engage FAK and its homolog Pyk2 (Mitra and Schlaepfer, 2006). Triple immunofluorescence confirmed that synaptic Act- $\beta 1$ is colocalized with FAK phosphorylated at its Tyr397 activation site (Fig. $1 B$ ). TBS produced a substantial increase in numbers of synapses containing Act- $\beta 1$ or pFAK at $2 \mathrm{~min}$, but not at $30 \mathrm{~s}$ or $7 \mathrm{~min}$, after stimulation (Fig. $1 C)$. TBS produced a slightly more protracted increase in activated pPyk 2 colocalized with PSD-95 (Fig. 1C).

Next, we tested whether the effects of TBS on FAK activation are dependent upon $\beta 1$ integrins by infusing neutralizing antisera to the $\beta 1$ integrin subunit at concentrations shown in previous studies to block both signaling initiated by integrin ligands in synaptoneurosomes (Bernard-Trifilo et al., 2005), and the contribution of $\beta 1$ integrins to LTP (Bernard-Trifilo et al., 2005; Kramár et al., 2006; Wang et al., 2008); anti-mouse IgG (IgG) was infused in control preparations. As in prior work (Kramár et al., 2006), $\beta 1$ neutralizing antisera did not influence baseline responses (fEPSP slope: + IgG $=98 \pm 2 \%$, + anti- $\beta 1=98 \pm 5 \%$, percentage of ACSFcontrol values) or short-term potentiation immediately after TBS (fEPSP slope $1 \mathrm{~min}$ post-TBS: $+\mathrm{IgG}=252 \pm 36 \%$, + anti- $\beta 1=$ $264 \pm 20 \%$; Fig. $1 D$ ). It did, however, completely block activity- integrins in adult hippocampus exhibit a refractory phenomenon that has not been reported for other types of adhesion junctions.

Next, we explored the possibility that integrin signaling becomes refractory to TBS2 applied during the $\sim 30$ min refractory period for integrin activation. As described, TBS1 increased the incidence of double-labeled ( $\mathrm{pFAK}+$ and PSD-95+) synapses in CA1b str. radiatum by $\sim 90 \%$ relative to values from control slices; TBS2 had little if any effect on synaptic pFAK when delivered $10(97 \pm 6 \%$ of control) or $30(124 \pm 12 \%)$ min later. The difference in TBS-induced pFAK + synapses in single vs repeated TBS cases was highly significant $(p<0.001$, ANOVA; $p<0.05$ for con vs $2 \mathrm{~min}, p<0.05$ for $2 \mathrm{~min}$ vs $10 \mathrm{~min}, \mathrm{~K}-\mathrm{W}$; Fig. $2 \mathrm{~B}$ ). The numbers of pFAK + puncta that were not associated with PSD-95 were comparable in control slices and those receiving two TBS trains ( $109 \pm 16 \%$ of control), indicating that the first TBS episode did not produce a generalized depression of pFAK immunolabeling. In contrast to effects of stimulation at shorter delays, TBS2 applied 60 min after TBS1 elicited a marked increase in pFAK + postsynaptic densities $(173 \pm 24 \%$ of control; $p<0.01$, two-tailed $t$ test) indicating that activity-induced integrin signaling was fully restored at this latency (Fig. $2 B$ ).

Pertinent to mechanisms underlying recovery, brefeldin A, which selectively suppresses endosome formation ( $\mathrm{Gu}$ et al., 2011) and integrin delivery to the plasma membrane in hip- 
pocampal neurons and other cell types (Lin et al., 2008), blocked integrin activation by TBS2 at $60 \mathrm{~min}$ post-TBS1, when infused starting $10 \mathrm{~min}$ after TBS1 (Fig. 2C). Brefeldin A pretreatment did not affect integrin activation by TBS1 (Fig. 2C), as expected given that integrins are already in place at this time. The TBS2specific effects of brefeldin A indicate that inactivation and insertion mechanisms regulating surface integrins in migrating cells (Gu et al., 2011) underlie the dynamics of synaptic integrins described here. Finally, TBS2-driven integrin activation seen at 60 min post-TBS1 was unaffected by infusion of the protein synthesis inhibitor anisomycin, indicating that the restoration of responsivity does not depend upon translation of new integrin proteins (Fig. 2D).

\section{Recovery of integrin responsiveness produces delayed consolidation of LTP}

The above findings raise the question of whether the loss and recovery of synaptic integrin signaling have functional consequences for LTP. Recovery could, for example, affect the constitutive contributions of integrins to maintaining cytoskeletal changes produced by TBS1. We investigated this possibility by infusing neutralizing anti- $\beta 1$, or control IgG, into field CA1b while recording fEPSPs. The control IgG had no effect on potentiated responses when infused starting at $25 \mathrm{~min}$ after theta stimulation. In contrast, with infusion of anti- $\beta 1$ initiated $25 \mathrm{~min}$ after TBS1, potentiation began to decay within $10 \mathrm{~min}$ and responses returned to baseline over the next $40 \mathrm{~min}$. This response to anti- $\beta 1$ was unexpected as it represents the only case, over a diverse array of manipulations (Lynch et al., 2007), in which we have been able to reverse LTP at $\sim 30 \mathrm{~min}$ after TBS. The effect of integrin neutralization was transient in that anti- $\beta 1$ had no effect on LTP when applied at $60 \mathrm{~min}$ post-TBS, when the integrin response to TBS was fully restored (Fig. $3 B$ ). Similar results were obtained with infusions of brefeldin A: applications starting 10 min post-TBS1 caused LTP to decay to baseline over the same time frame as seen with anti- $\beta 1$ treatment (Fig. $3 C$ ). We conclude that integrins contribute to a previously undetected phase of LTP consolidation that occurs 40-60 min after induction.

\section{Integrin antagonists applied during, but not after, recovery block memory formation}

Does the delayed phase of LTP stabilization contribute to the formation of long-term memory? We tested this using an object location memory task that is dependent upon rostral hippocampus (Barrett et al., 2011; Haettig et al., 2011). Following habituation to the apparatus, mice fitted with bilateral hippocampal cannulae were allowed to explore two identical objects in a 10 min training period (day 1) and then returned to the arena, with one object moved to a novel location, the following day (Fig. 4A). Trained mice spend more time exploring the novel-location object than the familiar one on day 2, indicating long-term memory for object location (Barrett et al., 2011). Either control IgG or neutralizing anti- $\beta 1$ (1.0 $\mu \mathrm{l}$ each) was infused bilaterally into rostral field CA1 starting 5, 20, or 60 min after training. Mice treated with the control IgG, at each of the three post-training time points, exhibited significant preference for the familiar object in the novel location on day 2 (DI: $17.48 \pm 5.61$ for $5 \mathrm{~min}$ group; $38.08 \pm 4.73$ for $20 \mathrm{~min}$ group; $23.64 \pm 6.15$ for $60 \mathrm{~min}$ group: $p<0.01$, one-sample $t$ test for each time point). In contrast, those injected with anti- $\beta 1$ at 5 or 20 min after training were completely amnestic for object location when tested on day 2 (DI: $-5.17 \pm 6.49$ and $4.06 \pm 4.71$, respectively); the differences in retention scores for IgG vs anti- $\beta 1$-treated mice were significant
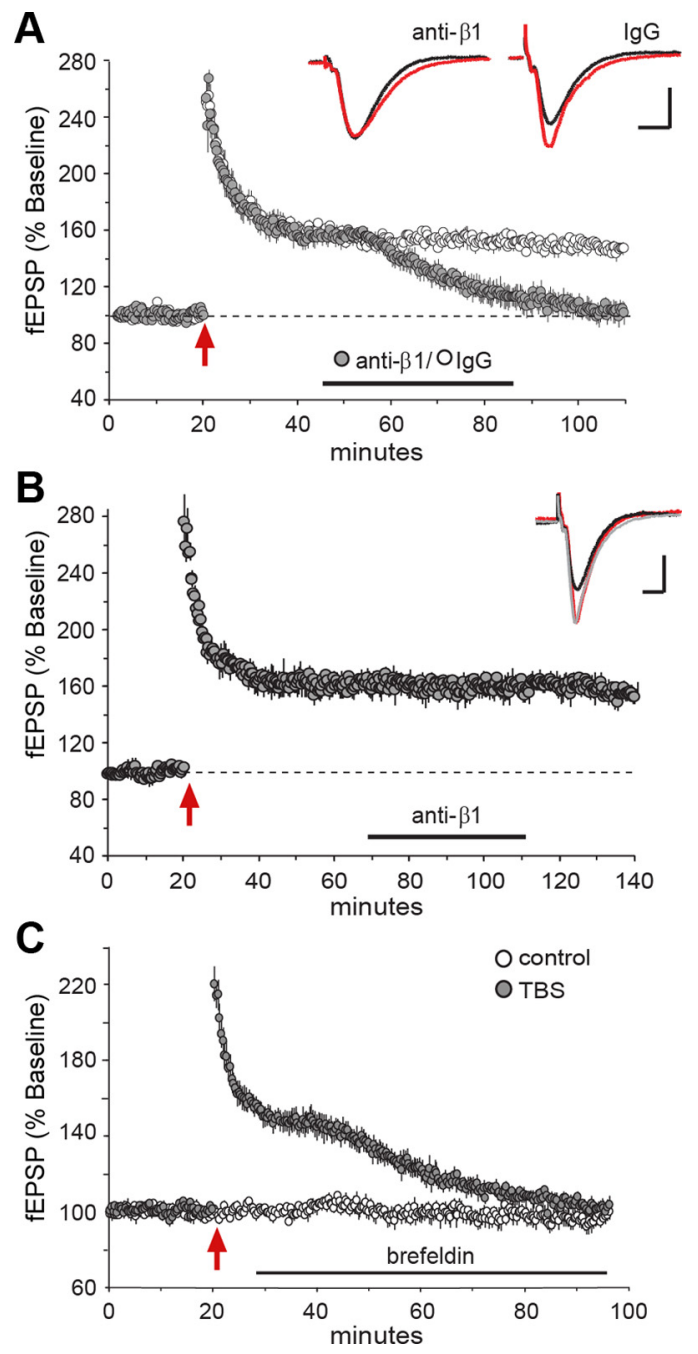

Figure 3. Integrin recovery is necessary for LTP consolidation. Hippocampal slices received stimulation of Schaffer-commissural projections and fEPSPs were recorded from CA1b str. radiatum; at different times following TBS (red arrow), neutralizing antisera to $\beta 1$ integrin (anti$\beta 1$ ) or brefeldin A were infused into the slice. $A$, Plot of fEPSPs shows that neutralizing anti- $\beta 1$ (closed circles), but not control lgG (open circles), destabilized LTP when applied to hippocampal slices during the recovery of integrin signaling (30-60 min post-TBS). Inset traces are fEPSPs recorded before (black) and 90 min after (red) TBS; waveforms after antibody-induced LTP reversal are the same as those for baseline responses (calibration: $1 \mathrm{mV}, 5 \mathrm{~ms}$ ). $\boldsymbol{B}$, Anti- $\beta 1$ had no effect on LTP when infused after integrin recovery was complete (starting 60 min postTBS). Traces show fEPSPs collected before TBS (black), 55 min after TBS (gray), and 120 min after TBS (red); those collected 55 and 120 min post-TBS are not detectably different (calibration: 1 $\mathrm{mV}, 5 \mathrm{~ms}$ ). C, Post-TBS infusion of brefeldin A (at horizontal bar, initiated $10 \mathrm{~min}$ after stimulation) caused a delayed decay of potentiation, similar to effects of anti- $\beta 1$ shown in $\boldsymbol{A}$.

for both the 5 and 20 min post-training infusion time points $(p<$ 0.01 and $p<0.0001$, respectively; Fig. $4 B$ ). These two groups did not differ in total time exploring objects $\left(t_{(17)}=-1.343, p=\right.$ $\left.0.200 ; t_{(18)}=-0.149, p=0.874\right)$ or for their discrimination index $\left(t_{(17)}=0.849, p=0.411 ; t_{(18)}=-0.149, p=0.874\right)$ during training. The dispersion of the infused antibody was verified by immunohistochemistry (Fig. 4C) and by significantly reduced numbers of pFAK + puncta in CA1b sample field $(35,151 \pm 790$ and 22,624 \pm 2942 mean puncta per sample field for IgG and anti- $\beta 1$ treatments, respectively; $p<0.01$, two-tailed $t$ test). Although somewhat surprising that treatments targeting a particular septo-temporal portion of hippocampus eliminate the formation of long-term memory, the result aligns with recent work showing that during unsupervised learning LTP-related actin signal- 
A

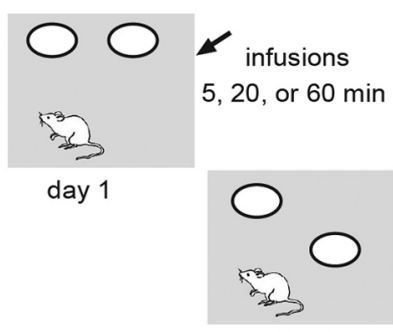

day 2
B

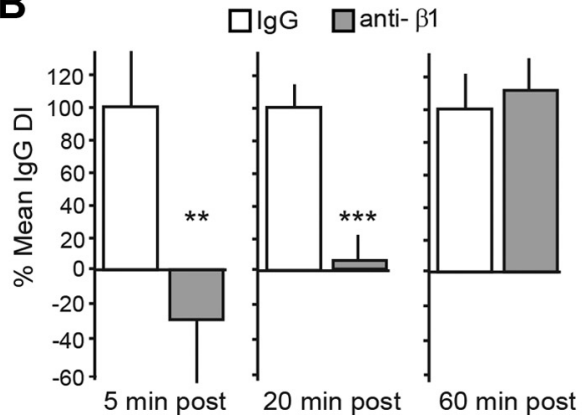

C

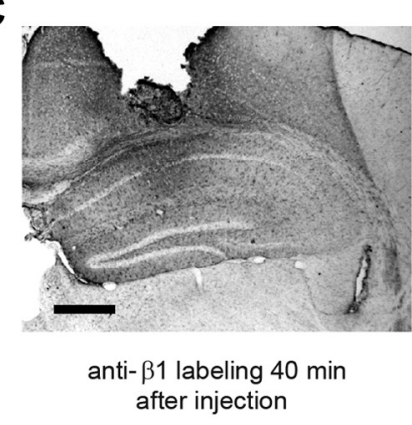

Figure 4. $\quad \beta 1$ integrins contribute to the stabilization of object location memory. $A$, Object location paradigm for studying long-term memory. Panel shows the open field apparatus with two open circles indicating the placement of identical objects; the location of one object is changed on day 2. B, Bar graphs show the discrimination indices of mice treated with neutralizing antisera to $\beta 1$-integrin (gray bars) expressed as a percentage of measures from paired lgG-treated controls (con; white bars); there were $8-10$ mice in each group. As shown, anti- $\beta 1$ infusion initiated 5 and 20 min after training reduced the time spent with the novel-location object on day 2 , whereas anti- $\beta 1$ infused 60 min after training had no effect $\left({ }^{* *} p<0.01\right.$; ${ }^{* * *} p<0.0001$ vs same time point IgG con, one-tailed $t$ test). C, Immunostaining for rabbit lgG shows the spread of the anti- $\beta 1$ in hippocampus for one case killed 40 min after infusion (using techniques used in behavioral studies); the antisera had clearly diffused throughout rostral hippocampus at this time point (scale bar, $0.5 \mathrm{~mm}$ ).

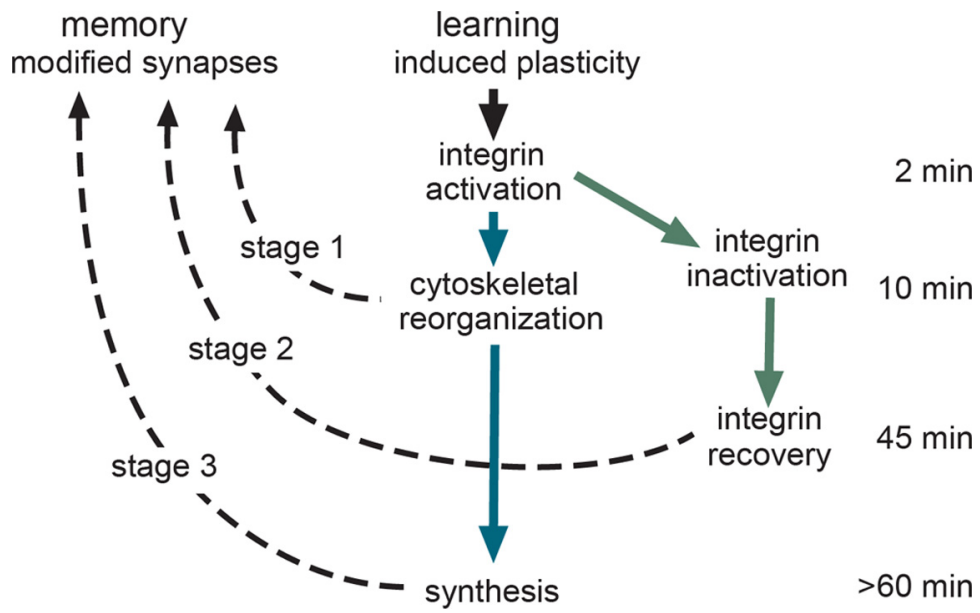

Figure 5. Model for multistage consolidation of synaptic plasticity and memory. Schematic showing the proposed serial stages in LTP and memory consolidation. Prior studies have shown that learning and LTP undergo rapid stabilization ( $\sim 10 \mathrm{~min})$ involving integrin-driven reorganization of the subsynaptic cytoskeleton (stage 1). The same events initiate local translation resulting in the later appearance of proteins needed for full stabilization of synaptic changes (stage 3). This constitutes a serial (early and late stages) consolidation pathway (blue arrows). The initial integrin activation is followed by inactivation and then, $\sim 45$ min later, "recovery" of responsivity. The latter event is also necessary for stabilization of synaptic changes, thus producing a parallel path (green arrows) leading to an intermediate stage of consolidation (stage 2).

ing within dendritic spines is also restricted to rostral hippocampal fields (Chen et al., 2010a,b). In contrast to the robust effects of anti- $\beta 1$ infusion at 5 and 20 min after training, anti- $\beta 1$ delivered 60 min after training had no effect on long-term memory of object location (DI = 26.55 \pm 6.37 ; Fig. $4 B$ ); thus, the time course for the delayed, integrin-dependent phase of LTP stabilization holds for memory encoding.

\section{Discussion}

The present findings provide direct evidence for stimulationinduced integrin activation and signaling at adult synapses. How theta bursts produce these effects remains to be determined although it is known that the stimulation pattern engages kinases and small GTPases (Chen et al., 2007, 2010b) that regulate integrin status in various types of adhesion junctions (Munshi and Stack, 2006; Jin et al., 2011; Sil et al., 2011). Moreover, recent studies showed that LTP induction leads to focal activation of matrix metalloproteinase-9 (MMP-9; Nagy et al., 2006; Bozdagi et al., 2007), an extracellular proteinase that cleaves matrix proteins and thereby generates integrin ligands (Wang et al., 2008). These experiments also established that MMP-9 activity leads to $\beta 1$ integrindependent phosphorylation of the actin severing protein cofilin, a result consonant with evidence that integrins are critical to TBSinduced actin polymerization (Kramár et al., 2006). This collection of findings provides an outline of mechanisms for producing the integrin activation that we have observed.

The results described here also show that at adult synapses integrins have dynamic properties, in particular very brief activation followed by a protracted loss of responsiveness, which could not have been predicted from prior work on synapses or other types of cellular junctions. It is of interest in this context that integrins are substrates for calcium-dependent proteases (calpains; Huttenlocher et al., 1997; Le Goff et al., 2010) that are activated within the spine compartment by TBS (del Cerro et al., 1990; Vanderklish et al., 2000; Lynch et al., 2007). The idea that proteolytic integrin inactivation may account for the post-TBS refractory period has the advantage of accounting for both the brevity of the initial synaptic integrin response (activation and signaling) and the need for an extended recovery period before TBS can again trigger integrin activation. This hypothesis would suggest that the latter refractory period reflects the time needed for transport and insertion of replacement integrin copies into synapses to restore integrin responsivity and signaling. Protease inhibitors are not sufficiently selective for a strong test of the inactivation component of the above argument. However, the fungal metabolite brefeldin A, which acutely blocks membrane insertion of integrins in dissociated cells and cultured hippocampal slices (Klausner et al., 1992; Lin et al., 2005), eliminated the delayed recovery of integrin responses to TBS, as predicted by the second part of the hypothesis. We therefore propose a working model in which theta bursts (1) activate synaptic integrins, (2) trigger inactivating proteolytic events, and (3) initiate cascades that replace the lost synaptic receptors.

Whatever its origins, the return of synaptic integrin responsiveness was shown to be critical for a previously undetected, 
delayed, and temporally limited phase of LTP consolidation. Neutralizing $\beta 1$ antisera, applied after the rapid consolidation of LTP (i.e., $\geq 10 \mathrm{~min}$ post-TBS), but before the onset of integrin recovery, produced a gradual, stereotyped reversal of potentiation; a comparable effect was obtained with infusion of brefeldin A. These are surprising results because LTP at $30 \mathrm{~min}$ post-TBS is resistant to a variety of manipulations (e.g., adenosine, lowfrequency stimulation, latrunculin, low temperature) that destabilize potentiation when applied shortly after induction (Lynch et al., 2007; Rex et al., 2010). In contrast, infusions of anti- $\beta 1$ had no effect on LTP when started $60 \mathrm{~min}$ after TBS, indicating that integrin recovery is required to initiate a delayed stage of consolidation but not to maintain the potentiated state. Past studies showed that TBS causes rapid ( $<2 \mathrm{~min}$ ), integrin-dependent formation of subsynaptic actin filaments (Kramár et al., 2006) that are stabilized by processes reflecting a second signaling cascade over the following 5-10 min (Rex et al., 2009). It is possible that the integrin recovery is needed to anchor the newly formed actin networks to the extracellular matrix, and thereby to maintain the structural modifications of synapses that accompany and stabilize LTP (Chen et al., 2007, 2010b; Yang et al., 2008; Bourne and Harris, 2012).

Tests for $\beta 1$-integrin involvement in phases of memory consolidation produced results comparable to those obtained in the LTP experiments: infusion of anti- $\beta 1$ into rostral hippocampus thoroughly blocked the formation of long-term object location memory when initiated up to 20 min after training but had no effect on next-day retention when begun $40 \mathrm{~min}$ later. Studies of conditional knock-outs (KOs) for hippocampal $\beta 1$ integrin expression (Chan et al., 2006) and of mutants with reduced expression of select $\alpha$ subunits that dimerize with $\beta 1$ (Chan et al., 2003, $2006,2007)$ reliably identify a role for $\beta 1$-family integrins in working memory but findings for long term memory (fear conditioning; spatial learning) have been mixed. In particular, long term spatial memory as tested in the Morris water maze was not disrupted by conditional deletion of $\beta 1$ (Chan et al., 2006) but was impaired by reduced expression of $\alpha$ subunits that exclusively dimerize with $\beta 1$ (Chan et al., 2003). The basis for these seemingly conflicting results is not known but recent evidence that conditional $\beta 1$ deletion is associated with increases in synaptic levels of select cell adhesion molecules (N-cadherin, neuroligins; Mortillo et al., 2012) suggests that interpretation of effects on learning in these mutants will not be straightforward. Given the selectivity of the experimental manipulation used in the present studies (neutralizing antisera), and its structural similarity to the control treatment (IgG), our behavioral results provide clear evidence for important $\beta 1$ integrin involvement in the consolidation of both LTP and hippocampus-dependent memory. More importantly, the present behavioral results point to integrin involvement in a previously undetected step in the sequence of events that transfers newly learned material into long term storage. A considerable body of work describes rapid stabilizing events occurring in the 5-10 min after learning (Lynch, 2004; Fedulov et al., 2007; Baudry et al., 2011), with much later processes, likely involving protein synthesis, needed to maintain the memory trace (Hernandez and Abel, 2008; Sacktor, 2008). The delayed, integrin-dependent modifications described here appear to constitute an intermediate stage that fits between the previously identified early and late phases of LTP and memory consolidation.

Interestingly, the postrefractory return of activity-induced integrin signaling demonstrated in the present hippocampal slice studies follows the same time course as the expression of a capac- ity for enhanced potentiation in response to successive theta trains (Kramár et al., 2012). In particular, we have shown that following an initial round of theta stimulation, a second bout of TBS applied 10 or 30 min later has no effect on the level of potentiation whereas the magnitude of LTP is significantly increased if TBS2 is applied $\geq 1 \mathrm{~h}$ after TBS1. Together these findings suggest the possibility that processes regulating the periodicity of integrin function may underlie a synaptic variant of the spaced trials effect wherein theta activity spaced by at least 60 min can elicit greater potentiation than can an equivalent amount of stimulation applied continuously, or with shorter intertrain intervals. Previous studies have suggested that the timing rules for spaced trials phenomena reflect the timing of memory trace consolidation (Wickelgren, 1974; Braun and Rubin, 1998). This idea fits with the present evidence that the return of integrin function is needed for both a delayed stage in LTP and memory consolidation and augmented plasticity in response to spaced theta trains.

An essential question in consolidation theory concerns the extent to which serial stages are causally linked or instead represent endpoints of parallel consolidation cascades initiated by learning (McGaugh, 2000). Our findings suggest a hybrid model (Fig. 5) wherein integrin activation is both necessary for the rapid consolidation of LTP and memory (Rex et al., 2009, 2010), and sets in motion delayed integrin-related changes required for further stabilization. There are also results indicating that the rapid cytoskeletal modifications driven in part by synaptic integrins contribute importantly to local protein synthesis and thus to a still later stage of consolidation (Vanderklish et al., 2000; Bramham, 2008; Sacktor, 2008). We therefore propose that rapid consolidation is linked to both intermediate and late consolidation, and that the latter two are not themselves connected (Fig. 5).

\section{References}

Barrett RM, Malvaez M, Kramar E, Matheos DP, Arrizon A, Cabrera SM, Lynch G, Greene RW, Wood MA (2011) Hippocampal focal knockout of CBP affects specific histone modifications, long-term potentiation, and long-term memory. Neuropsychopharmacology 36:1545-1556.

Baudry M, Bi X, Gall C, Lynch G (2011) The biochemistry of memory: the 26 year journey of a 'new and specific hypothesis'. Neurobiol Learn Mem 95:125-133.

Bernard-Trifilo JA, Kramár EA, Torp R, Lin CY, Pineda EA, Lynch G, Gall CM (2005) Integrin signaling cascades are operational in adult hippocampal synapses and modulate NMDA receptor physiology. J Neurochem 93:834-849.

Bourne JN, Harris KM (2012) Nanoscale analysis of structural synaptic plasticity. Curr Opin Neurobiol 22:372-382.

Bozdagi O, Nagy V, Kwei KT, Huntley GW (2007) In vivo roles for matrix metalloproteinase-9 in mature hippocampal synaptic physiology and plasticity. J Neurophysiol 98:334-344.

Brakebusch C, Fässler R (2003) The integrin-actin connection, an eternal love affair. EMBO J 22:2324-2333.

Bramham CR (2008) Local protein synthesis, actin dynamics, and LTP consolidation. Curr Opin Neurobiol 18:524-531.

Braun K, Rubin DC (1998) The spacing effect depends on an encoding deficit, retrieval, and time in working memory: evidence from oncepresented words. Memory 6:37-65.

Chan CS, Weeber EJ, Kurup S, Sweatt JD, Davis RL (2003) Integrin requirement for hippocampal synaptic plasticity and spatial memory. J Neurosci 23:7107-7116.

Chan CS, Weeber EJ, Zong L, Fuchs E, Sweatt JD, Davis RL (2006) Beta 1 -integrins are required for hippocampal AMPA receptor-dependent synaptic transmission, synaptic plasticity, and working memory. J Neurosci 26:223-232.

Chan CS, Levenson JM, Mukhopadhyay PS, Zong L, Bradley A, Sweatt JD, Davis RL (2007) Alpha3-integrins are required for hippocampal longterm potentiation and working memory. Learn Mem 14:606-615. 
Chan CS, Chen H, Bradley A, Dragatsis I, Rosenmund C, Davis RL (2010) $\alpha 8$-integrins are required for hippocampal long-term potentiation but not for hippocampal-dependent learning. Genes Brain Behav 9:402-410.

Chen LY, Rex CS, Casale MS, Gall CM, Lynch G (2007) Changes in synaptic morphology accompany actin signaling during LTP. J Neurosci 27:5363-5372.

Chen LY, Rex CS, Pham DT, Lynch G, Gall CM (2010a) BDNF signaling during learning is regionally differentiated within hippocampus. J Neurosci 30:15097-15101.

Chen LY, Rex CS, Sanaiha Y, Lynch G, Gall CM (2010b) Learning induces neurotrophin signaling at hippocampal synapses. Proc Natl Acad Sci U S A 107:7030-7035.

Chen LY, Rex CS, Babayan AH, Kramár EA, Lynch G, Gall CM, Lauterborn JC (2010c) Physiological activation of synaptic Rac $>$ PAK (p-21 activated kinase) signaling is defective in a mouse model of fragile $\mathrm{X}$ syndrome. J Neurosci 30:10977-10984.

Chun D, Gall CM, Bi X, Lynch G (2001) Evidence that integrins contribute to multiple stages in the consolidation of long term potentiation in rat hippocampus. Neuroscience 105:815-829.

del Cerro S, Larson J, Oliver MW, Lynch G (1990) Development of hippocampal long-term potentiation is reduced by recently introduced calpain inhibitors. Brain Res 530:91-95.

Fedulov V, Rex CS, Simmons DA, Palmer L, Gall CM, Lynch G (2007) Evidence that long-term potentiation occurs within individual hippocampal synapses during learning. J Neurosci 27:8031-8039.

Fukazawa Y, Saitoh Y, Ozawa F, Ohta Y, Mizuno K, Inokuchi K (2003) Hippocampal LTP is accompanied by enhanced F-actin content within the dendritic spine that is essential for late LTP maintenance in vivo. Neuron 38:447-460.

Gu Z, Noss EH, Hsu VW, Brenner MB (2011) Integrins traffic rapidly via circular dorsal ruffles and macropinocytosis during stimulated cell migration. J Cell Biol 193:61-70.

Haettig J, Stefanko DP, Multani ML, Figueroa DX, McQuown SC, Wood MA (2011) HDAC inhibition modulates hippocampus-dependent longterm memory for object location in a CBP-dependent manner. Learn Mem 18:71-79.

Hernandez PJ, Abel T (2008) The role of protein synthesis in memory consolidation: progress amid decades of debate. Neurobiol Learn Mem 89:293-311.

Huang Z, Shimazu K, Woo NH, Zang K, Müller U, Lu B, Reichardt LF (2006) Distinct roles of the beta 1-class integrins at the developing and the mature hippocampal excitatory synapse. J Neurosci 26:11208-11219.

Huttenlocher A, Palecek SP, Lu Q, Zhang W, Mellgren RL, Lauffenburger DA, Ginsberg MH, Horwitz AF (1997) Regulation of cell migration by the calcium-dependent protease calpain. J Biol Chem 272:32719-32722.

Jin YJ, Park I, Hong IK, Byun HJ, Choi J, Kim YM, Lee H (2011) Fibronectin and vitronectin induce AP-1-mediated matrix metalloproteinase- 9 expression through integrin alpha(5)beta(1)/alpha(v)beta(3)-dependent Akt, ERK and JNK signaling pathways in human umbilical vein endothelial cells. Cell Signal 23:125-134.

Klausner RD, Donaldson JG, Lippincott-Schwartz J (1992) Brefeldin A: insights into the control of membrane traffic and organelle structure. J Cell Biol 116:1071-1080.

Kramár EA, Bernard JA, Gall CM, Lynch G (2002) Alpha3 integrin receptors contribute to the consolidation of long-term potentiation. Neuroscience 110:29-39.

Kramár EA, Lin B, Rex CS, Gall CM, Lynch G (2006) Integrin-driven actin polymerization consolidates long-term potentiation. Proc Natl Acad Sci U S A 103:5579-5584.

Kramár EA, Babayan AH, Gavin CF, Cox CD, Jafari M, Gall CM, Rumbaugh G, Lynch G (2012) Synaptic evidence for the efficacy of spaced learning. Proc Natl Acad Sci U S A 109:5121-5126.

Krucker T, Siggins GR, Halpain S (2000) Dynamic actin filaments are required for stable long-term potentiation (LTP) in area CAl of the hippocampus. Proc Natl Acad Sci U S A 97:6856-6861.

Lamprecht R (2011) The roles of the actin cytoskeleton in fear memory formation. Front Behav Neurosci 5:39.

Legate KR, Fässler R (2009) Mechanisms that regulate adaptor binding to beta-integrin cytoplasmic tails. J Cell Sci 122:187-198.

Le Goff E, Vallentin A, Harmand PO, Aldrian-Herrada G, Rebière B, Roy C, Benyamin Y, Lebart MC (2010) Characterization of L-plastin interac- tion with beta integrin and its regulation by micro-calpain. Cytoskeleton 67:286-296.

Lin CY, Lynch G, Gall CM (2005) AMPA receptor stimulation increases $\alpha 5 \beta 1$ integrin surface expression, adhesive function and signaling. J Neurochem 94:531-546.

Lin CY, Hilgenberg LG, Smith MA, Lynch G, Gall CM (2008) Integrin regulation of cytoplasmic calcium in excitatory neurons depends upon glutamate receptors and release from intracellular stores. Mol Cell Neurosci $37: 770-780$.

Luque A, Gómez M, Puzon W, Takada Y, Sánchez-Madrid F, Cabañas C (1996) Activated conformations of very late activation integrins detected by a group of antibodies (HUTS) specific for a novel regulatory region (355-425) of the common beta 1 chain. J Biol Chem 271:11067-11075.

Lynch G, Rex CS, Gall CM (2007) LTP consolidation: substrates, explanatory power, and functional significance. Neuropharmacology 52:12-23.

Lynch MA (2004) Long-term potentiation and memory. Physiol Rev 84:87-136.

McGaugh JL (2000) Memory-a century of consolidation. Science 287:248-251

Mitra SK, Schlaepfer DD (2006) Integrin-regulated FAK-Src signaling in normal and cancer cells. Curr Opin Cell Biol 18:516-523.

Mortillo S, Elste A, Ge Y, Patil SB, Hsiao K, Huntley GW, Davis RL, Benson DL (2012) Compensatory redistribution of neuroligins and N-cadherin following deletion of synaptic beta1-integrin. J Comp Neurol 520:2041-2052.

Motanis H, Maroun M (2012) Differential involvement of protein synthesis and actin rearrangement in the reacquisition of contextual fear conditioning. Hippocampus 22:494-500.

Müller GE, Pilzecker A (1900) Experimentelle Beiträge zur Lehre vom Gedächtnis. Z Psychol S 1:1-288.

Munshi HG, Stack MS (2006) Reciprocal interactions between adhesion receptor signaling and MMP regulation. Cancer Metastasis Rev 25:45-56.

Nagy V, Bozdagi O, Matynia A, Balcerzyk M, Okulski P, Dzwonek J, Costa RM, Silva AJ, Kaczmarek L, Huntley GW (2006) Matrix metalloproteinase-9 is required for hippocampal late-phase long-term potentiation and memory. J Neurosci 26:1923-1934.

Oinuma I, Katoh H, Negishi M (2006) Semaphorin 4D/Plexin-B1-mediated R-Ras GAP activity inhibits cell migration by regulating beta(1) integrin activity. J Cell Biol 173:601-613.

Rex CS, Chen LY, Sharma A, Liu J, Babayan AH, Gall CM, Lynch G (2009) Different Rho GTPase-dependent signaling pathways initiate sequential steps in the consolidation of long-term potentiation. J Cell Biol 186:85-97.

Rex CS, Gavin CF, Rubio MD, Kramar EA, Chen LY, Jia Y, Huganir RL, Muzyczka N, Gall CM, Miller CA, Lynch G, Rumbaugh G (2010) Myosin IIb regulates actin dynamics during synaptic plasticity and memory formation. Neuron 67:603-617.

Ribot T (1882) Diseases of memory. New York: Appleton.

Sacktor TC (2008) PKMzeta, LTP maintenance, and the dynamic molecular biology of memory storage. Prog Brain Res 169:27-40.

Scales TM, Parsons M (2011) Spatial and temporal regulation of integrin signalling during cell migration. Curr Opin Cell Biol 23:562-568.

Sil H, Sen T, Chatterjee A (2011) Fibronectin-integrin (alpha5betal) modulates migration and invasion of murine melanoma cell line B16F10 by involving MMP-9. Oncol Res 19:335-348.

Vanderklish PW, Krushel LA, Holst BH, Gally JA, Crossin KL, Edelman GM (2000) Marking synaptic activity in dendritic spines with a calpain substrate exhibiting fluorescence resonance energy transfer. Proc Natl Acad Sci U S A 97:2253-2258.

Vecsey CG, Hawk JD, Lattal KM, Stein JM, Fabian SA, Attner MA, Cabrera SM, McDonough CB, Brindle PK, Abel T, Wood MA (2007) Histone deacetylase inhibitors enhance memory and synaptic plasticity via CREB: CBP-dependent transcriptional activation. J Neurosci 27:6128-6140.

Wang XB, Bozdagi O, Nikitczuk JS, Zhai ZW, Zhou Q, Huntley GW (2008) Extracellular proteolysis by matrix metalloproteinase- 9 drives dendritic spine enlargement and long-term potentiation coordinately. Proc Natl Acad Sci U S A 105:19520-19525.

Wickelgren WA (1974) Single-trace fragility theory of memory dynamics. Mem Cognit 2:775-780.

Yang Y, Wang XB, Frerking M, Zhou Q (2008) Spine expansion and stabilization associated with long-term potentiation. J Neurosci 28:57405751 . 